\title{
Self-Assembly of a Water Chain with Tetrameric and Decameric Clusters in the Channel of a Mixed-Valence $\mathrm{Cu}^{\mathrm{I}} \mathrm{Cu}^{\mathrm{II}} \mathrm{Complex}$
}

\author{
Ning-Hai Hu, ${ }^{*}$ Zhi-Gang Li, Jing-Wei Xu, ${ }^{*}$ Heng-Qing Jia, and Jia-Jia Niu \\ Changchun Institute of Applied Chemistry, Chinese Academy of Sciences, Changchun, 130022, \\ P. R. China. Fax: 86431 5685653.E-mail: hunh@ciac.jl.cn
}

Supporting Information

Table S1. Hydrogen bond lengths and angles in $\mathbf{1 .}$

\begin{tabular}{|c|c|c|c|c|c|}
\hline $\mathrm{D}-\mathrm{H} \ldots \mathrm{A}^{a}$ & $\mathrm{D}-\mathrm{H}(\AA ̊)$ & $\mathrm{H} \ldots \mathrm{A}(\AA)$ & $D \ldots A(\AA ̊)$ & $\mathrm{D}-\mathrm{H}-\mathrm{A}\left({ }^{\circ}\right)$ & $\begin{array}{l}\text { Symmetry } \\
\text { operation for } \mathrm{A}\end{array}$ \\
\hline $\mathrm{O} 1 \mathrm{w}-\mathrm{H} 1 \mathrm{a} \ldots \mathrm{...} 2 \mathrm{w}$ & 0.93 & 2.10 & $2.954(4)$ & 152 & $x, y, z$ \\
\hline O1w-H1b...O5w & 0.95 & 2.04 & $2.958(4)$ & 162 & $1-x, 1-y,-z$ \\
\hline O2w-H2a...O3 & 0.95 & 1.81 & $2.751(3)$ & 169 & $x, y, z$ \\
\hline $\mathrm{O} 2 \mathrm{w}-\mathrm{H} 2 \mathrm{~b} \ldots \mathrm{O} 4 \mathrm{w}$ & 1.05 & 1.71 & $2.746(3)$ & 167 & $1-x, 1-y,-z$ \\
\hline О3w-H3a...О5w & 0.92 & 1.99 & $2.887(4)$ & 166 & $x, y, z$ \\
\hline $\mathrm{O} 3 w-\mathrm{H} 3 b . . . \mathrm{O} 2 \mathrm{w}$ & 0.89 & 1.94 & $2.782(4)$ & 156 & $x, y, z$ \\
\hline $\mathrm{O} 4 \mathrm{w}-\mathrm{H} 4 \mathrm{a} \ldots . . \mathrm{O} 3 \mathrm{w}$ & 1.01 & 1.85 & $2.783(4)$ & 151 & $x, y, z$ \\
\hline $\mathrm{O} 4 \mathrm{w}-\mathrm{H} 4 \mathrm{~b} \ldots \mathrm{O} 1 \mathrm{w}$ & 0.99 & 1.89 & $2.871(4)$ & 172 & $x, y, z$ \\
\hline O5w-H5a...O2 & 0.90 & 1.97 & $2.797(3)$ & 153 & $-1+x, y, z$ \\
\hline O5w-H5b...O6w & 0.87 & 1.92 & $2.791(3)$ & 176 & $x, y, z$ \\
\hline O6w-H6a...O4 & 0.90 & 1.88 & $2.764(3)$ & 166 & $x, y, z$ \\
\hline O6w-H6b...O7w & 1.00 & 1.83 & $2.812(3)$ & 167 & $x, y, z$ \\
\hline O7w-H7a...O2 & 0.89 & 1.86 & $2.729(3)$ & 165 & $-1+x, y, z$ \\
\hline O7w-H7b...O6w & 1.02 & 1.81 & $2.820(4)$ & 170 & $1-x,-y, 1-z$ \\
\hline
\end{tabular}

${ }^{a} \mathrm{D}=$ donor atom, $\mathrm{A}=$ acceptor atom. 


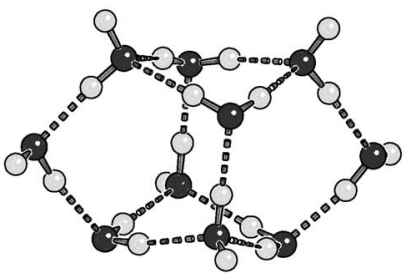

(a)

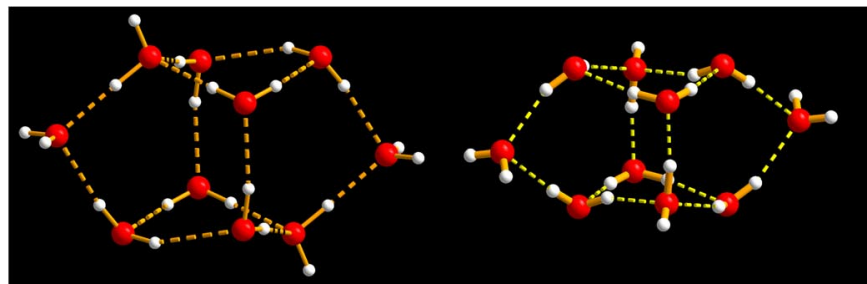

(b) (c)

Figure S1. Comparison of hydrogen-bonding schemes in the water decamers from Buck's model (a)(Buck, U.; Ettischer, I.; Melzer, M.; Buch, V.; Sadlej, J. Phys. Rev. Lett. 1998, 80, 2578), reported by Ermer and Neudörfl (b) (Ermer, O.; Neudörfl, J. Chem. Eur. J. 2001, 7, 4961) and observed in 1 (c).

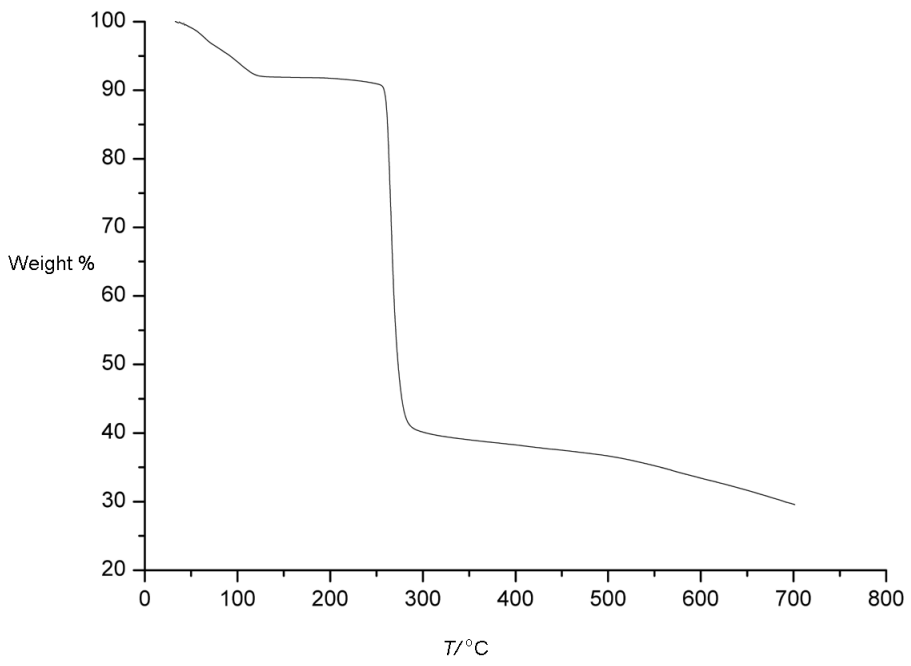

Figure S2. Thermogravimetric curve for 1. 
a)

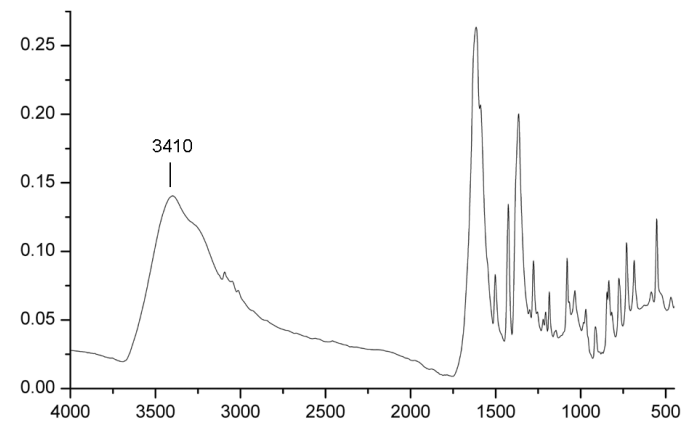

b)

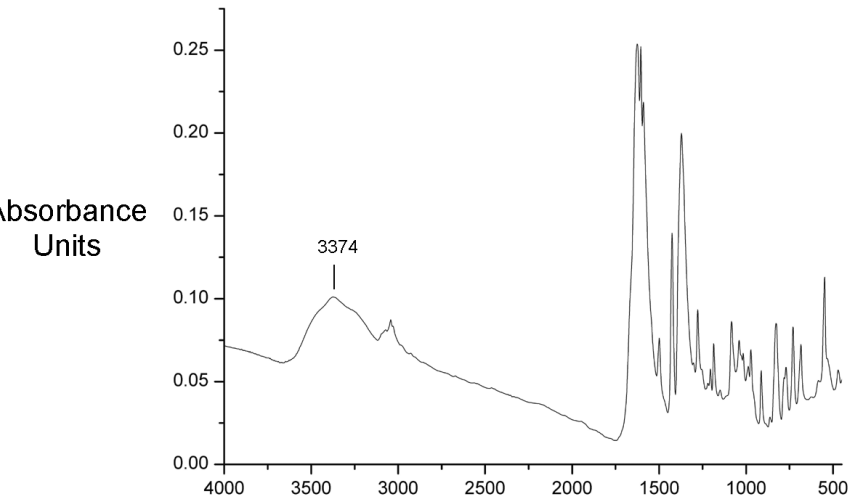

c)

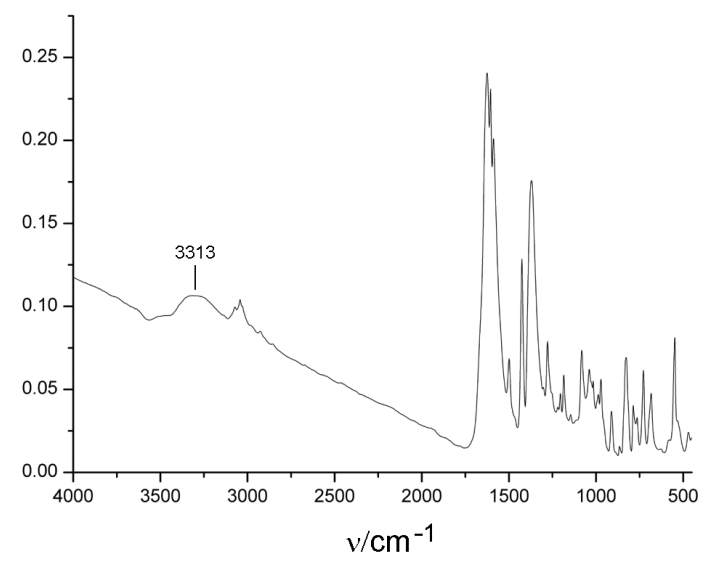

Figure S3. The FT-IR spectra of $\mathbf{1}$ (in $\mathrm{KBr}$ disc) before (a) and after heating at $100{ }^{\circ} \mathrm{C}$ for $5 \mathrm{~h} \mathrm{(b)} \mathrm{and} 7 \mathrm{~h} \mathrm{(c)}$. 

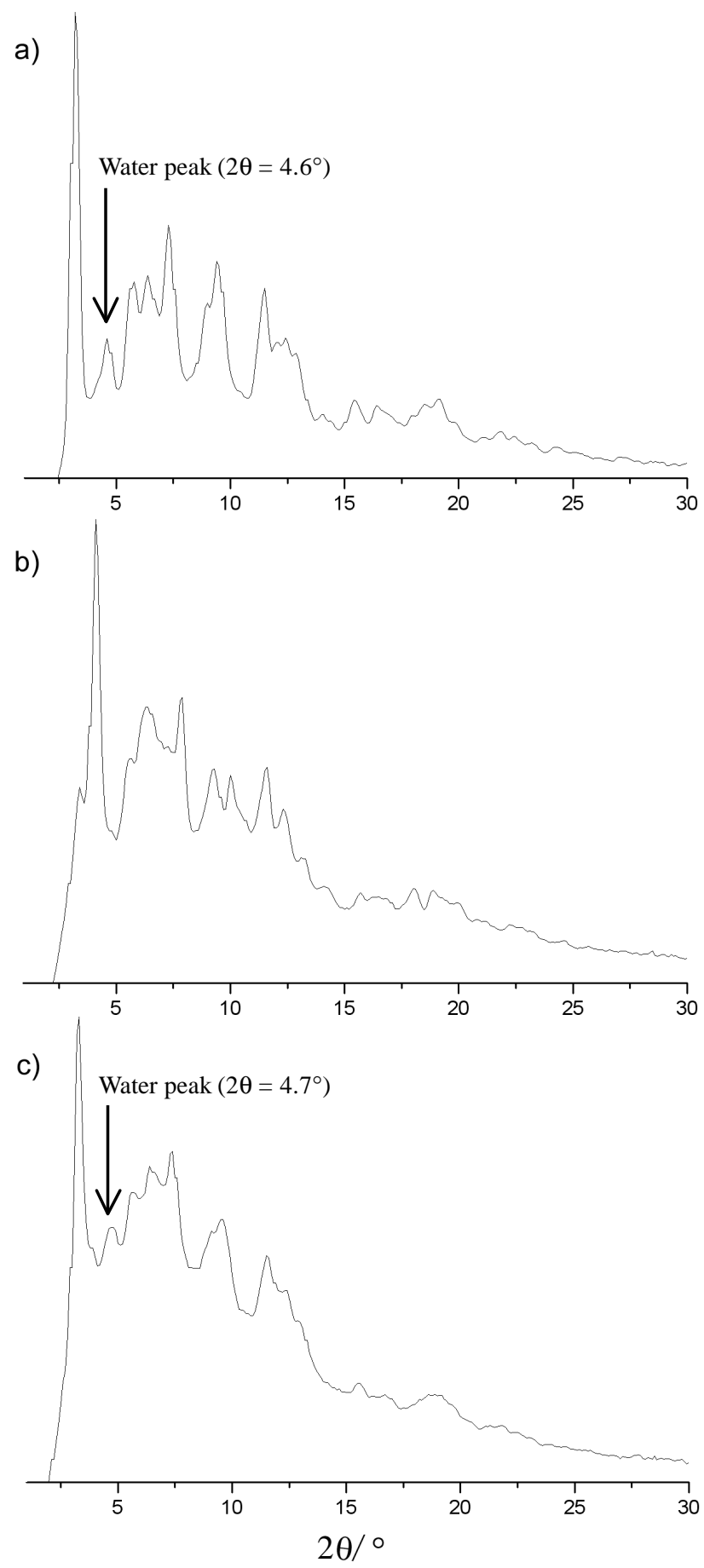

Figure S4. The X-ray powder diffraction patterns of 1 (MoK $\alpha$ radiation, $\lambda=0.71073 \AA$ ), as-synthesized (a), after dehydration at $110{ }^{\circ} \mathrm{C}$ for $5 \mathrm{~h} \mathrm{(b),}$ and after rehydration in humidity (c). 
a)

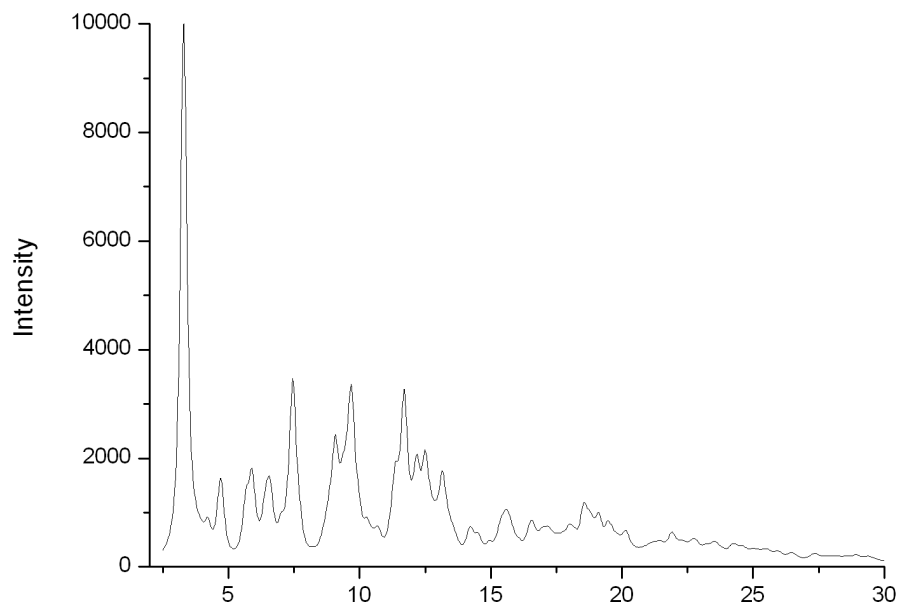

b)

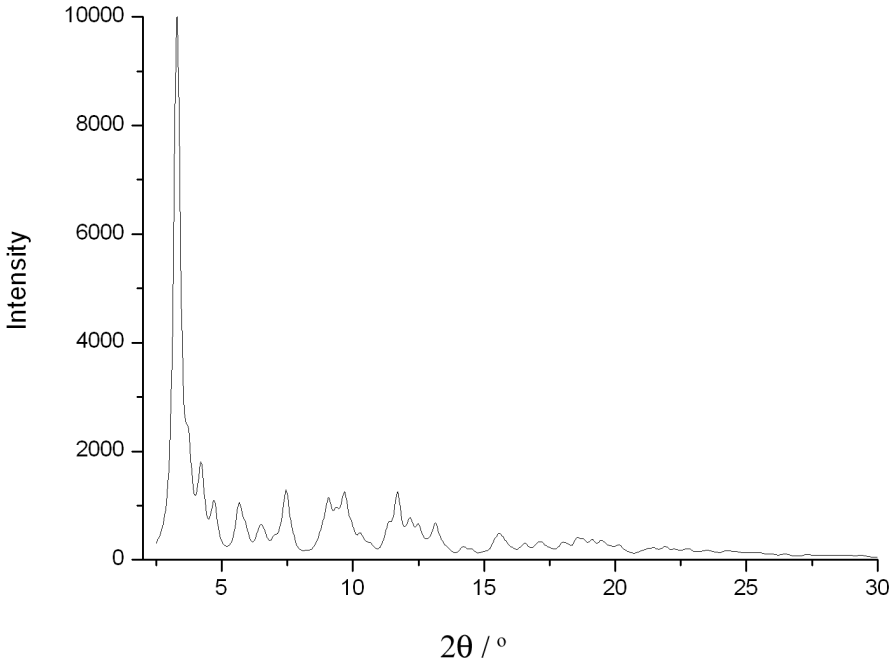

Figure S5. The simulated XRPD patterns $(\lambda=0.71073 \AA)$ of $\mathbf{1}$ (a), and after the water molecules were omitted (b). 